\title{
Adverse Course of Listeria monocytogenes Infection in Pediatric Patient with T-Cell Acute Lymphoblastic Leukemia
}

\begin{abstract}
Adamczewska-Wawrzynowicz K, Płotka A*, Wziątek A, Wachowiak J and Derwich $K$

Department of Pediatric Oncology, Hematology and Transplantology, Poznań University of Medical Sciences, Poznań, Poland

*Corresponding author: Płotka A. Student's Scientific Circle at Department of Pediatric Oncology, Hematology and Transplantology, Poznań University of Medical Sciences, ul. Szpitalna 27/33, 60-572 Poznań, Poland
\end{abstract}

Received: January 21, 2019; Accepted: February 25, 2019; Published: March 04, 2019

\begin{abstract}
Oncological patients are at an increased risk of an opportunistic infection caused by Listeria monocytogenes. Listeriosis remains infrequent in oncological patients. This article describes a case report of a 15 -year old boy diagnosed with T-ALL, who developed L.monocytogenes infection during an intensive cancer treatment. The patient's treatment proceeded with numerous complications. Impaired coagulation led to genetic examination and factor $\mathrm{V}$ Leiden and heterozygotic C667T and A1298C mutations of MTHFR detection. Corticosteroid therapy resulted in insulin resistance and hyperbilirubinemia with normal hepatic enzymes level. Gilbert's syndrome was confirmed. CT evaluating remission revealed asymptomatic invasive pulmonary aspergillosis. After finishing Protocol II the rise in CRP was observed with decreased number of WBC. Patient was feverish and complained of nonspecific abdominal pain and dysuria. USG detected fluid in right iliac fossa. A few hours later boy's neurological state dramatically worsened. CT revealed acute hydrocephalus, which needed immediate drainage. Listeria monocytogenes was isolated from CSF. Despite intensive antibiotic therapy, infection caused boy's death.
\end{abstract}

Keywords: Acute lymphoblastic leukemia; Opportunistic infection; Listeria monocytogenes; Pediatric oncology

\section{Case Presentation}

15-year-old boy was referred to Department of Pediatric Oncology, Hematology and Transplantology with suspicion of Non-Hodgkin Lymphoma (NHL). Patient's history revealed musculoskeletal pain, emesis and epistaxis. On clinical examination massive submandibular and cervical lymphadenopathy, petechiae all over the body, especially severe in pelvic girdle, splenomegaly (spleen reaching lower quadrant) and hepatomegaly $(3 \mathrm{~cm}$ above subcostal arch), tachycardia (150/min), on auscultation diminished vesicular murmur paraspinal on both sides were noticed. Blood tests revealed leukocytosis (104 ths./ $\mu \mathrm{l})$, III grade thrombocytopenia (22ths/ $\mu \mathrm{l})$ and hyperuricemia $(27 \mathrm{mg} / \mathrm{dl})$, high level of creatinine $(1,18 \mathrm{mg} / \mathrm{dl})$ and urea $(75 \mathrm{mg} / \mathrm{dl})$. Boy presented with, both laboratorary and clinically, renal insufficiency in $4^{\text {th }}$ stage (GFR-22 $\mathrm{ml} / \mathrm{min}$ ).

Intravenous rehydration, rasburicase and diuretics were administered resulting in gradual decrease and normalisation of uric acid. RTG and CT examinations revealed widened mediastinum (implying tumour), pleural and pericardial effusion. Due to clinical presentation and laboratory findings, theinitialdiagnosis of T-cellacute lymphoblastic leukemia was made and confirmed by myelogram and immunological examination of bone marrow. Treatment according to ALL IC BFM 2009 for intermediate-risk ALL was immediately administered. During induction phase clinical and biochemical signs of renal insufficiency occurred. Patient required hemodialysis with calcium supplementation $(\mathrm{Ca}-0,81 \mathrm{mmol} / \mathrm{L})$ and hyperphosphatemia management at the same time $(\mathrm{P}-12,42 \mathrm{mmol} / \mathrm{L})$. Boy developed symptoms of tetany, which disappeared after normalisation of calcium level. Because of coagulation disorders genetic examinations were carried out resulting in diagnosis of Leiden and heterozigotic C667T and A1298C mutation of MTHFR. Induction glucocorticosteroid therapy triggered decrease glucose-tolerance with insulin resistance. During diabetic diet hyperbilirubinemia $(4,41 \mathrm{mg} \%)$ with normal liver blood tests was observed, suggesting Gilbert syndrome, confirmed later by genetic examination. Long-term steroids administration caused adrenal cortical insufficiency, presenting with hyperhidrosis, alabaster-coloured pale skin, muscle weakness, fatigue and polyuria. Patient also developed symptoms of secondary hypopituitarism with hypothyroidism, which required hydrocortisone and levothyroxine supplementation. CT scan, carried out to asses remission of leukemia, revealed invasive fungal infection, despite the lack of any clinical or laboratory signs. Basing on bronchoalveolar lavage examination Aspergillus fumigatus and CMV infections was diagnosed. After 14 days of combined treatment (voriconazole, itraconazole,acyclovir) regression of radiological abnormalities was seen. After patient's clinical improvement, induction (Protocole I) and consolidation (Protocole M) chemotherapy was continued without complications. During reinduction (Protocole II) patient needed insulinotherapy, plasma transfusions and antithrombin supplementation. High dosages of glicocorticosteroids triggered hyperglycemia and behavioural disorders and needed to be reduced. Continous hyponatremia and hyperpotassemia due to lack of minercorticoids led to need of fludrocortisone supplementation. At the end of Protocole II CRP was gradually increasing with decreasing level of leukocytes at the same time $(250 / \mu \mathrm{l})$. Patient developed fever and complained of unspecific abdominal pain, straining to defecate and dysuria.
Ann Hematol Oncol - Volume 6 Issue 3 - 2019

ISSN : 2375-7965 | www.austinpublishing group.com

Płotka et al. (C) All rights are reserved
Citation: Adamczewska-Wawrzynowicz K, Płotka A, Wziątek A, Wachowiak J and Derwich K. Adverse Course of Listeria monocytogenes Infection in Pediatric Patient with T-Cell Acute Lymphoblastic Leukemia. Ann Hematol Oncol. 2019; 6(3): 1240. 
He was given empirical antibiotics (piperacillin, tazobactam and amikacin) and rutine bacterial cultures were collected. Abdominal ultrasonography detected free fluid in right iliac fossa. On $4^{\text {th }}$ day of antibiotic treatment patient's medical condition dramatically worsened. The level of fluid increased, suggesting appendicitis. Patient was qualified to diagnostic laparoscopy. Neurological state deterioration (asymetric pupils, nuchal rigidity) began on the same day. Emergent head CT scan confirmed acute hydrocephalus, which required urgent external ventricular drainage Listeria monocytogenes was isolated from both blood and cerebrospinal fluid. In spite of immediate amikacin (500mg) and meropenem $(40 \mathrm{mg} / \mathrm{kg}$ ) infusion boy's medical state drastically decreased, resulting in patient's death.

\section{Discussion}

This article describes a case report of a patient diagnosed with T-ALL, who developed L.monocytogenes infection during an intensive cancer treatment. We would like to emphasize the diagnostic difficulties and a dramatic course of the opportunistic infection.

Acute Lymphoblastic Leukemia (ALL) is the most prevalent childhood malignancy. It comprises about $30 \%$ of all neoplastic diseases among children [1]. T-cell ALL (T-ALL) is estimated to comprise $10-15 \%$ of cases [2]. In recent years the overall survival among children with ALL has been documented as increasing. Nevertheless, despite intensification of the therapy, failure of treatment is observed in about $20-25 \%$ of patients [3]. Infections remain the most frequent complication of antineoplastic treatment and are associated with a high risk of mortality [4]. One of the pathogens responsible for opportunistic infections is Listeria monocytogenes (L.monocytogenes). Oncological patients are considered to be at an increased risk of L.monocytogenes infections [5]. A supreme prognostic factor in T-ALL treatment is the Prednisone Response (PR). The connection between PR and positive outcomes of the therapy has been proved [6]. However, despite ascertained Prednisone-Good Response (PGR) on the $8^{\text {th }}$ day of the therapy and confirmed Complete Remission (CR) on the $15^{\text {th }}$ and $33^{\text {rd }}$ day, the patient's treatment proceeded with complications.

Signs of an Invasive Pulmonary Aspergillosis [IPA] on the chest CT scan were an accidental discovery. An invasive aspergillosis is one of the most frequent and most dangerous opportunistic infection among immunocompromised patients [7]. Aspergillus grows extremely rapidly in a lung tissue and the infection is initially asymptomatic. A non-specific chest pain may be the first sign of an IPA. Most often, the only perceptible sign of evolving pulmonary fungal infection is a persistent Febrile Neutropenia (FN) despite broad-spectrum antibiotics. Signs as cough, chest pain, pleural rubs or hemoptysis emerge gradually with the development of the disease. Nevertheless, patients who receive corticosteroids may not develop fever, as in the case of our patient. It is recommended to perform a high-resolution CT scan of the chest to detect signs of an IPA in highrisk patients with a persistent FN between $3^{\text {rd }}$ and $7^{\text {th }}$ day of the FN. It is a valuable test to identify the halo sign, an early sign of an IPA with a short duration [8]. We would like to emphasize that our patient did not present with any signs of an IPA. The CT scan, performed to evaluate remission of mediastinal masses, revealed the IPA signs accidentally.
Despite an effective antifungal therapy and, above all, good response to the antileukemic treatment confirmed by complete remission, the boy died due to meningitis caused by L.monocytogenes. The correlation between neoplastic diseases and listeriosis was reported in 1967 for the first time [5]. Despite an increased predisposition for infection, listeriosis remains infrequent in oncological patients. The low incidence of a disseminated infection may be a result of an antimicrobial prophylaxis (trimethoprim-sulfamethoxazole, TMP-SMX) administered as a routine to immunocompromised patients. TMP-SMX has a proven efficacy in the prevention of an opportunistic infection-Pneumocystis Jirovecii Pneumonia (PJP) in immunodeficient patients [9]. The above-mentioned drug was administered to our patient in compliance with the ALL IC BFM 2009 protocol. According to numerous reports in the literature, TMPSMX has even broader spectrum of activity. In recent years it has been found to be a protective agent against listeriosis, legionellosis, toxoplasmosis and salmonellosis in immunocompromised patients [10]. It is worth mentioning that the implemented prophylaxis did not protected the boy from the infection. Despite numerous studies on efficiency of TMP-SMX, cases of listeriosis in oncological patients receiving TMP-SMX has also been reported. [11] Patients with Listeria meningitis usually present with: movement disorders (ataxia, tremors, myoclonus), seizures and fluctuating mental status [12]. Psychological disorders may precede other symptoms of the infection by a week or more. Episodes of stupor may alternate with intervals of normal state of consciousness. Nagging but rather mild apathy may suggest only fatigue, depression or side effects of sedative drugs. Aberrant hallucinations or illusions may also occur [13]. Comparable symptoms have been observed in our patient. The boy presented with depressed mood, apathy and abnormal behaviour repeatedly during hospitalization a few weeks prior to an exacerbation of neurological disorders. On the basis of the psychiatric consultation psychological care was implemented. The markers of an inflammation-CRP increase, fever, decreased leukocytes-were observed only after certain time. Non-specific abdominal pain, dysuria and fluid in the right iliac fossa were suggestive of an appendicitis. Administration of an empiric antibiotic therapy for febrile neutropenia-piperacillin/ tazobactam and amikacin-was ineffectual. In case of suspicion of L.monocytogenes meningitis ampicillin remains a treatment of choice [14]. In our patient, however, no signs of L.monocytogenes meningitis were reported at the beginning. Hence, the boy did not receive this therapy. Only significant exacerbation of patient's neurological condition, anisocoria and stiffness of neck suggested diagnosis. The final diagnosis was made on a basis of a microbiological investigation of Cerebrospinal Fluid (CSF) from which L.monocytogenes was isolated. An acute hydrocephalus, revealed in the cranial CT scan, is a rare complication of Listeria meningitis. Targeted antibiotic therapy-ampicillin with gentamicin-and prompt cerebrospinal fluid drainage may achieve full recovery [15]. Despite therapy, mortality rate among patients due to L.monocytogenes CNS infection is high, reaching up to $50 \%$ [16-20]. The insidious course of the infection and late onset of neurological symptoms were responsible for the delay in the diagnosis. Hence, prompt administration of the targeted therapy was impossible. To conclude, during intensive ALL treatment possibility of numerous early complications leading to chemotherapy delay and resulting in decreased effectiveness must by remembered. Immunological state and additional diseases of our patients may be 
the background for severe opportunistic infections, becoming even the cause of sudden death.

\section{References}

1. Lo Nigro. Biology of childhood acute lymphoblastic leukemia. J Pediatr Hematol Oncol. 2013; 35: 245-252.

2. Karman K, Johansson B. Pediatric T-cell acute lymphoblastic leukemia. Genes Chromosomes Cancer. 2017; 56: 89-116.

3. Uckun FM, Sensel MG, Sun L, Steinherz PG, Trigg ME, Heerema NA, et al. Biology and Treatment of Childhood T-Lineage Acute Lymphoblastic Leukemia. Blood. 1998; 91: 735-746.

4. Hughes WT, Armstrong D, Bodey GP, Bow EJ, Brown AE, Calandra T, et al. 2002 guidelines for the use of antimicrobial agents in neutropenic patients with cancer. Clin Infect Dis. 2002; 34: 730-751.

5. Louria DB, Hensle T, Armstrong D, et al. Listeriosis complicating malignant disease: a new association. Ann Intern Med. 1967; 67: 261-281.

6. Schrappe M, Möricke A, Reiter A, Henze G, Welte K, Gadner H, et al. Key treatment questions in childhood acute lymphoblastic leukemia: results in 5 censecutive trials performed by the ALL-BFM study group from 1981 to 2000 . Klin Padiatr. 2013; 225: 62-72.

7. Gallien S, Fournier S, Porcher R, Bottero J, Ribaud P, Sulahian A, et al Therapeutic Outcome and Prognostic Factors of Invasive Aspergillosis in an Infectious Disease Department: A Review of 34 Cases. Infection. 2008; 36 533-538

8. Reichenberger F, Habicht JM, Gratwohl A, Tamm M. Diagnosis and treatment of invasive pulmonary aspergillosis in neutropenic patients. Eur Respir J. 2002; 19: 743-755.

9. Hughes WT, Rivera GK, Schell MJ, Thornton D, Lott L. Successful intermittent chemoprophylaxis for Pneumocystis carinii pneumonitis. N Eng J Med. 1987; 316: 1627-1632

10. Fernàndez-Sabé N, Cervera $C$, López-Medrano $F$, Llano $M$, Sáez E, Len Ó, et al. Risk factors, clinical features and outcomes of listeriosis in solid-organ transplant recipients: a matched case-control study. Clin Infect Dis. 2009; 49: 1153-1159.
11. Bodro M, Paterson DL. Has the Time Come for Routine TrimethoprimSulfamethoxazole Prophylaxis in Patients Taking Biologic Therapies? Clin Infect Dis. 2013; 56: 1621-1628.

12. Tsai SH, Chu SJ, Wu CP, Wang NC. Listerial meningitis in a patient with undiagnosed acquired immunodeficiency syndrome: ampicillin should be added to the empirical antibiotic coverage. Emerg Med J. 2006; 23: e50.

13. J Simpson. Listeria monocytogenes meningitis: an opportunistic infection. J Neurolo Neurosurg Psychiatry. 1971; 34: 657-663.

14. Mylonakis E, Hohmann EL, Calderwood SB. Central nervous system infection with Listeria monocytogenes. 33 years' experience at a general hospital and review of 776 episodes from the literature. Medicine (Baltimore). 1998; 77: 313-336.

15. Yang CC, Yeh $\mathrm{CH}$, Tsai TC, Yu WLJ. Acute symptomatic hydrocephalus in Listeria monocytogenes meningitis. Microbiol Immunol Infect. 2006; 39: 255258.

16. Kołakowska A, Madajczak G. Pałeczki Listeria monocytogenes w zakażeniach ludzi. Przegl Epidemiol. 2011; 65: 57-62.

17. R Rozen. Genetic predisposition to hyperhomocysteinemia: deficiency of Methylenetetrahydrofolate Reductase (MTHFR). Thromb Haemost. 1997; 78: 523-526.

18. MM Jadaon. Epidemiology of Activated Protein C Resistance and Factor $V$ Leiden Mutation in the Mediterranean Region. Mediterr J Hematol Infect Dis. 2011; 3: e2011037.

19. Gollan JL, Bateman C, Billing BH. Effect of dietary composition on the unconjugated hyperbilirubinaemia of Gilbert's syndrome. Gut. 1976; 17: 335340 .

20. Esbenshade AJ, Simmons JH, Koyama T, Lindell RB, Friedman DL. Obesity and insulin resistance in pediatric acute lymphoblastic leukemia worsens during maintenance therapy. Pediatr Blood Cancer. 2013; 60: 1287-1291.
Ann Hematol Oncol - Volume 6 Issue 3 - 2019

ISSN : 2375-7965 | www.austinpublishing group.com

Płotka et al. (C) All rights are reserved
Citation: Adamczewska-Wawrzynowicz K, Płotka A, Wziątek A, Wachowiak J and Derwich K. Adverse Course of Listeria monocytogenes Infection in Pediatric Patient with T-Cell Acute Lymphoblastic Leukemia. Ann Hematol Oncol. 2019; 6(3): 1240 